\section{The importance of relaxation}

\author{
Myron Lecar
}

Dynamical Evolution of Globular Clusters. By Lyman Spitzer, Jr. Princeton University Press:1988. Pp.180. Hbk \$35, $£ 20.50$; pbk $\$ 14.50, £ 8.50$.

Our Galaxy contains close to 200 globular clusters. A variety of age indicators (chemical composition, orbits and the age of the oldest stars still on the main sequence) confirm that they were born early in the life of the Galaxy, about $10^{10}$ years ago. The study of the dynamical evolution of these clusters is irresistible to statistical physicists because this age is much longer than their 'relaxation time' (that is, the time for the orbits of individual stars to be appreciably perturbed by two-body encounters). Most of the well-observed globular clusters have relaxation times of between $10^{8}$ and $10^{10}$ years. By comparison, the relaxation time of the Galaxy exceeds $10^{14}$ years. The present structure of most globular clusters should show few traces of their initial structure.

In much the same way as stars, globular clusters evolve through a sequence of quasi-steady states, which are steady state solutions of the collisionless Boltzmann equation. There is no equilibrium state; the system always increases its entropy by forming a tightly bound core (to increase the negative binding energy) while expanding a halo or unbinding stars (to occupy a larger volume in phase space). But, because the time for a star to orbit the cluster is much shorter than the relaxation time, the structure is almost stationary on a dynamical time scale.

For most of their lives, the evolution of globular clusters is well traced by solutions of the Fokker-Planck equation. Professor Spitzer has been a leader in the simulation of solutions to the Fokker-Planck equation by Monte-Carlo techniques. In this book, he discusses his own methods and results, and gives fair attention to the other major practitioners of the MonteCarlo technique (Henon, Shapiro and Teukolsky) and to the direct solution of the Fokker-Planck equation by Cohn.

Spitzer's skilful illumination of the physical phenomena that drive evolution means that this book will become a classic. Using idealized models, he illustrates the phenomena of evaporation of stars from an isolated cluster; from a tidally limited cluster; mass stratification; and the collapse of the isothermal core because of the gravo-thermal instability. Heating of the halo by compressive shocks (as the cluster passes through the disk of the galaxy) and tidal shocks are also treated.
The book concludes with a discussion of the current puzzle in globular cluster evolution. It is now firmly believed that the evolution proceeds to a thermal runaway in the core. After a finite time, the density of an innermost region, well within the isothermal core, tends to infinity. Furthermore, many globular clusters should have undergone such core collapse some time ago. Theorists are now asking: "What happens after core collapse?". A plausible suggestion is that when the density becomes very high, binary stars are formed which, through close encounters with a third star, increase the kinetic energy of the core, at the expense of increasing the (negative) binding energy of the binary. This process is another example, on a smaller scale, of the same instability that prompted the core collapse in the first place. The influx of kinetic energy into the core brakes the collapse of the core and causes it to expand. Some simulations indicate a cycle of collapse followed by re-expansion.

These phenomena are currently being studied. For an astrophysicist who wants to join the endeavour, Spitzer's book will be indispensable. Here is to be found a complex theoretical structure assembled by a master craftsman. The writing style is compact, which probably makes the book unsuitable for an introductory course in stellar dynamics, but this is an excellent text for a graduate course or seminar.

Myron Lecar is at the Harvard-Smithsonian Center for Astrophysics, 60 Garden Street, Cambridge, Massachusetts 02138, USA.

\section{Getting results}

\section{F.W. Bullock}

How Experiments End. By Peter Galison. University of Chicago Press: 1988. Pp.330. Hbk £31.95, \$47.95; pbk£11.95, \$17.95.

A Discussion of Einstein's work as an experimental physicist is hard to come by. For instance, Ronald W. Clark in his biography makes no mention of the Einstein/ de Haas experiments which were aimed at determining the $g$-factor of the electron. That these took place at about the same time as Einstein was struggling with the General Theory of Relativity, and was anxiously (or confidently perhaps) awaiting the outcome of the ill-fated Freundlich solar eclipse expedition to the Crimea, would appear to testify to the range of Einstein's abilities and interests.

It is more likely, however, that Einstein was not an active participant at the laboratory bench in the experiments which form part of the first case study in Galison's exhaustive search for the factors determining at what point an experimental study can be said to have achieved its objectives and can therefore be concluded. The other two series of experiments analysed in impressive detail are those (mainly cosmic-ray) experiments which eventually led to the discovery of the muon, and the accelerator experiments which resulted in the discovery of the neutral current process in weak interactions. In each of these studies the author presents the many levels of theoretical supposition that determined the experimenters' activities in deciding both the objectives of their experiments and the way the data were analysed and eventually presented.

The depth of Galison's research cannot be denied. In the case of the discovery of the weak neutral current he has been able to interview many of the researchers involved. Although his account would not always agree in fine detail with every memory of those who participated in the experiments, there is no doubt that he has understood very well the factors which determined events, from the initial speculation that an effect might be present to the final publication reporting the existence of neutral currents.

Some 80 per cent of the book is taken up by a comprehensive and scholarly account of theories, apparatus, experimental method and, in some cases, the personalities and prejudices of the people involved. Although providing ample evidence of the author's industry, and some interesting reading, it is often difficult to perceive the relevance of all the minutiae included. Indeed, on reaching the final chapters the reader may well be anticipating that his reward for persevering will be the appearance of a philosophical statement of some depth. In this respect, the conclusion is anti-climactic. Much of what has appeared before is repeated and the attempt to parse the events into a braudelian-type structure of long-term, medium-term and short-term constraints does not illuminate in any novel way the question of how experiments end.

In spite of this, any scientist interested in the philosophy or sociology of the experimental method will find the book worth reading. It demonstrates not only the many achievements of scientific research but also the many failings of those who pursue it. At the end, however, one must still wonder not so much how or why experiments end, but why the author chose these particular experiments to analyse. He gives us no reason to believe that they are typical of experimental physics and certainly leaves open the question of whether they are in any way representative of science in general.

F.W. Bullock is a Reader in the Department of Physics and Astronomy, University College London, Gower Street, London WC1E 6BT, UK. 